\title{
Second Order Receptive Field Properties of Simple and Complex Cells Support a New Standard Model of Thalamocortical Circuitry in V1
}

\author{
Madineh Sedigh-Sarvestani, Ivan Fernandez-Lamo, Andrew Jaegle, and M. Morgan Taylor \\ Department of Neuroscience, Perelman School of Medicine, University of Pennsylvania, Philadelphia, Pennsylvania 19104 \\ Review of Fournier et al.
}

Hubel and Wiesel's (1962) hierarchical model of simple and complex cell receptive fields (RFs) has shaped our understanding of the structure and function of the primary visual cortex (V1). They proposed that the characteristic elongated ON or OFF subregions of simple-cell RFs are constructed from the spatial alignment of lateral geniculate cells. In addition, the ON-OFF regions of complexcell RFs may arise from the alignment of spatially offset iso-oriented simple cells.

Simple-cell activity is usually described by LNP models (linear-nonlinear-Poisson), where the stimulus is filtered by a single linear spatiotemporal filter, then rectified by a fixed nonlinearity, and passed through a Poisson process to generate spikes. The linear spatiotemporal filter is usually estimated by reverse correlation of the stimulus pattern on spikes to obtain a spike-triggered average (STA). STA approaches are not suitable for estimation of linear filters in complex cells because the linear filters of these cells have similar responses to bright and dark stimuli, which cancel on average. Accordingly, complex cells have historically been modeled using "motion energy" approaches that predict firing rate

Received June 13, 2014; accepted July 12, 2014.

Correspondence should be addressed to Madineh Sedigh-Sarvestani,

121 Anatomy-Chemistry Building, University of Pennsylvania, Philadelphia, Pennsylvania 19104. E-mail: madineh@upenn.edu.

DOI:10.1523/JNEUROSCI.2425-14.2014

Copyright $\odot 2014$ the authors $\quad 0270-6474 / 14 / 3411177-03 \$ 15.00 / 0$ by squaring the output of two linear filters with similar feature selectivity.

These canonical models reflect two inherent dichotomies: the anatomical distinction between thalamic and intracortical inputs, and the functional distinction between linear and nonlinear stimulusresponse relationships. It has proven difficult to directly compare the RFs of simple and complex cells given the differences between these types of models.

A more recent approach combines STA with spike-triggered covariance (STC) analysis, which uses reverse correlation to characterize the second-order nonlinear (covariance) properties of the spike-triggered stimuli. Just as STA finds the mean stimulus that precedes a spike, STC estimates the covariance among stimulus dimensions that precede a spike (the spike-triggered stimulus set). The STC matrix is high dimensional and includes many features that do not affect the response of the cell. To estimate the relevant features, eigenvalues of the STC matrix are compared with null eigenvalues of the covariance matrix of the entire stimulus set. Eigenvectors of the STC matrix whose associated eigenvalues are significantly greater than those of the null set represent stimulus features (subunits) that increase the response of the cell, whereas eigenvectors with significantly smaller eigenvalues correspond to subunits that suppress the response. These subunits reflect the balance of excitation and inhibition. For example, a reduction of excitation or an increase in inhibition can both give rise to suppressive subunits. Each subunit contributes to the cell's response according to an estimated weight, which can be confirmed by playing these subunits back to the cell. Just as the STA subunit captures the aggregate input processed linearly by the cell, STC subunits capture the aggregate input that is processed nonlinearly. A subunit does not necessarily reflect a specific thalamic or cortical input.

Several investigators have applied STC methods to V1 (Touryan et al., 2002; Rust et al., 2005; Chen et al., 2007). Their results generally indicate that simple and complex cells are not purely linear and nonlinear operators but instead form a continuum between these extremes. A new standard model is being developed that accounts for linear and nonlinear relationships and can be used to model both cell types. The structural implication is that simple and complex cells receive both thalamic and intracortical inputs, albeit in different amounts, and do not form a simple hierarchy. An important shortcoming of these studies is the dependence of robust RF characterization on spike count. Using spikes, it is impossible to determine whether the set of subunits reflects the full selectivity of the neuron or whether this set excludes subunits that elicit only weak spiking responses. 


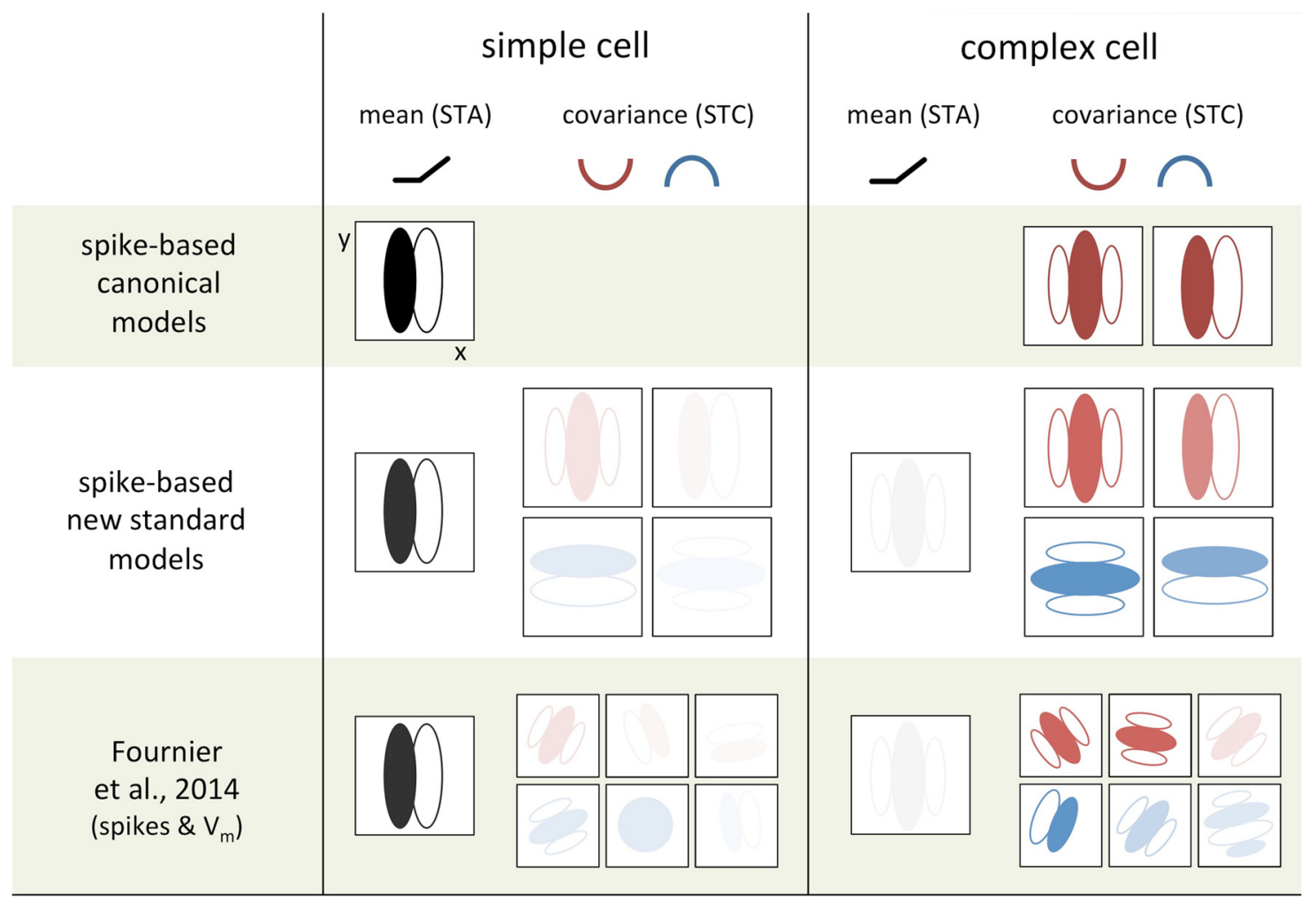

Figure 1. Predictions of the canonical and new standard models of simple and complex cell receptive fields in V1. The canonical model predicts one excitatory STA subunit (greyscale) for simple cells and a pair of excitatory covariance subunits (red) for complex cells. The newer models recovered both excitatory and suppressive (blue) covariance subunits for simple cells, although their contribution was less than the STA subunit (relative weights reflected by color intensity). Complex cells also exhibited more subunits than predicted by the canonical model, including a weak STA subunit. The orientation preference of the suppressive subunits was found to be opposite to the excitatory subunits. Fournier et al. (2014) also found additional subunits for simple and complex cells. However, they found a wide range of orientation preference across subunits.

Fournier et al. (2014) extend these STC-based approaches while circumventing the spike count shortcoming by using intracellular measurements of subthreshold neuronal responses. This methodology allows them to recover twice as many covariance subunits (referred to as complex-like subunits) at the synaptic level than at spiking level. The synaptic subunits contribute to spiking output according to their weights, with the most strongly weighted synaptic subunits corresponding to the spiking subunits. Their results indicate that intracellular measurements can be used to improve the characterization of relevant RF properties.

Although the use of subthreshold measurements introduces additional mathematical complexity, the design of this study is essentially the same as that previously conducted on the spiking output of V1 cells in the macaque (Rust et al., 2005; Chen et al., 2007). Similar to these studies, Fournier et al. (2014) found more subunits in both simple and complex cells than predicted by canonical models (Fig. 1). They found that the distinction between simple and complex cells is not in the number of STA versus covariance subunits, but rather in their relative weights.
Simple-cell RFs reflect a stronger contribution of the STA subunit, whereas complex-cell RFs reflect a greater contribution of the covariance subunits. They conclude that simple and complex cell characteristics alike arise due to different balances of thalamic and intracortical inputs, which exhibit linear and nonlinear stimulus-response relationships, respectively.

A notable distinction in Fournier et al.'s (2014) findings is the feature selectivity of the excitatory and suppressive covariance subunits. Previous studies (Rust et al., 2005; Chen et al., 2007) found that while excitatory covariance subunits were tuned to the same direction as the STA subunit, suppressive subunits were tuned to the opposite direction. A similar relationship was found in the orientation domain (Chen et al., 2007). These studies implied that suppressive subunits may sharpen feature selectivity by reducing the response to antagonistic features. In contrast, Fournier et al. (2014) generally found covariance subunits with a wide variety of orientation preferences and with no systematic relationship with the STA subunit preference (Fournier et al., 2014, their Fig. 7). The authors consider this mixture of orientation preference as sup- porting evidence that some covariance subunits reflect lateral connections between cortical columns.

Another new finding is a substantial number of suppressive covariance subunits that exhibit no orientation selectivity, corresponding to spatial RFs with broad inhibitory regions. Notably, these untuned subunits appear more frequently in simple cells (Fournier et al., 2014, their Fig. 4). As the authors point out, nonoriented inhibition may play a role in contrast invariance of V1 cell selectivity and other properties not accounted for by isooriented push-pull type inhibition, such as divisive gain control. It is unclear whether the discrepancies between Fournier et al. (2014) and prior studies arise due to species or methodological differences.

Verification of these membrane potential covariance-based results with another measure of synaptic input such as conductance may help to clarify these findings. In addition, conductance-based analyses can distinguish between changes in the balance of excitation and inhibition, which is important for defining the synaptic basis of subunits revealed by covariance-based analysis. The handful of conductance studies that directly measure 
orientation tuning of excitatory and inhibitory synaptic inputs have produced mixed results, but the current evidence seems to favor iso-oriented excitation and inhibition in both simple and complex cells (Anderson et al., 2000; Tan et al., 2011). There is no conductance-based evidence to support untuned inhibition in cat V1, although some studies discarded cells with untuned conductances. Further studies are needed to align conductance- and covariance-based measures of synaptic input.

Aside from cat and macaque, mice are becoming a popular species for the study of visual processing due to available genetic manipulations. Conductance studies have revealed fundamental differences between inhibition in the mouse and cat (Tan et al., 2011). Although inhibition appears to be iso-tuned with excitation in the mouse, there is currently no evidence to support asynchronous push-pull inhibition in this species. Thus it would be informative to repeat this analysis in the mouse and compare conductance- and covariance-based measures of synaptic input across species. Another informative experiment in the mouse would be to compare fast-spiking putatively inhibitory interneurons (FS) and regularspiking putatively excitatory cells (RS). Mouse FS cells receive stronger and more numerous projections from the thalamus than RS cells (Cruikshank et al., 2007), and should therefore reveal a greater ratio of STA-to-covariance subunit weight than RS cells.

The main hypothesis of Fournier et al. (2014), also proposed by others, is that simple and complex cells arise due to different balances of thalamic and intracortical input. This framework predicts that simple and complex cells, regardless of their laminar location, should exhibit some stimulus-dependent RF properties, such as linearity of spatial summation. Indeed, increased RF linearization as a function of spatiotemporal stimulus properties has been observed in cat V1 (Fournier et al., 2011) as well as in rat barrel cortex (Ramirez et al., 2014). Studies that simultaneously measure thalamic and intracortical input are necessary to better understand the effect of various spatiotemporal stimulus distributions on thalamocortical and intracortical drive. Nonetheless, the cautionary message is that RF properties estimated from one stimulus type may not be appropriate for prediction of responses to a different stimulus. This may explain the inability of the new standard models, estimated from white noise, to reliably predict neuronal responses to natural stimuli.

\section{References}

Anderson JS, Carandini M, Ferster D (2000) Orientation tuning of input conductance, excitation, and inhibition in cat primary visual cortex. J Neurophysiol 84:909-926. Medline
Chen X, Han F, Poo MM, Dan Y (2007) Excitatory and suppressive receptive field subunits in awake monkey primary visual cortex. Proc Natl Acad Sci USA 104:19120-19125. CrossRef Medline

Cruikshank SJ, Lewis TJ, Connors BW (2007) Synaptic basis for intense thalamocortical activation of feedforward inhibitory cells in neocortex. Nat Neurosci 10:462-468. CrossRef Medline

Fournier J, Monier C, Pananceau M, Frégnac Y (2011) Adaptation of the simple or complex nature of V1 receptive fields to visual statistics. Nat Neurosci 14:1053-1060. CrossRef Medline

Fournier J, Monier C, Levy M, Marre O, Sári K, Kisvárday ZF, Frégnac Y (2014) Hidden complexity of synaptic receptive fields in cat $\mathrm{V} 1$. J Neurosci 34:5515-5528. CrossRef Medline

Hubel DH, Wiesel TN (1962) Receptive fields, binocular interaction and functional architecture in the cat's visual cortex. J Physiol 160: 106-154. Medline

Ramirez A, Pnevmatikakis EA, Merel J, Paninski L, Miller KD, Bruno RM (2014) Spatiotemporal receptive fields of barrel cortex reveled by reverse correlation of synaptic input. Nat Neurosci 17:866-875. CrossRef Medline

Rust NC, Schwartz O, Movshon JA, Simoncelli EP (2005) Spatiotemporal elements of macaque V1 receptive fields. Neuron 46:945-956. CrossRef Medline

Tan AY, Brown BD, Scholl B, Mohanty D, Priebe NJ (2011) Orientation selectivity of synaptic input to neurons in mouse and cat primary visual cortex. J Neurosci 31:12339-12350. CrossRef Medline

Touryan J, Lau B, Dan Y (2002) Isolation of relevant visual features from random stimuli for cortical complex cells. J Neurosci 22:1081110818. Medline 